\title{
Detection of formimino-glutamic and urocanic acids in urine by means of thin-layer chromatography
}

\author{
MICHAEL ROBERTS ANDS. D. MOHAMED \\ From the Department of Materia Medica and Therapeutics, \\ University of Aberdeen, and the Royal Infirmary, Aberdeen
}

SYNOPSIS A simple and rapid method has been developed for the simultaneous separation and semi-quantitative estimation of formimino-glutamic and urocanic acids in urine. The urinary excretion of these compounds in a group of normal subjects and in a number of illustrative cases has been determined. In view of current opinion that it is the total of these two metabolites of histidine which is the significant figure in clinical investigation, their simultaneous separation and measurement is an important advantage of the method.

Deficiency of the folic acid coenzymes required in the final stages of histidine breakdown to glutamic acid (Fig. 1) may lead to increased urinary excretion of formimino-glutamic acid (FIGLU) (Bakerman, Silverman, and Daft, 1951; Borek and Waelsch, 1953). Estimation of urinary FIGLU has, therefore, become accepted clinical practice for the evaluation of megaloblastic anaemias in man. A variety of methods, quantitative and semi-quantitative, has been recommended. These include electrophoresis by low (Kohn, Mollin, and Rosenbach, 1961) and high (Knowles, Prankerd, and Westall, 1960) voltage; paper chromatography (Luhby, Cooperman, and Teller, 1959a, 1959b); and enzymatic (Tabor, and Wyngarden, 1958) and microbiological (Silverman, Gardiner, and Condit, 1958) assays. The sensitivity of the methods has been increased by preliminary histidine loading (Tabor, Silverman, Mehler, Daft, and Bauer, 1953).

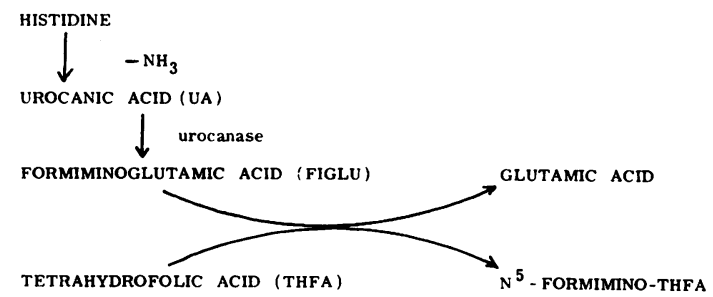

FIG. 1. Pathway of histidine metabolism.

Bennett and Chanarin (1962) have recently emphasized the need for simultaneous estimation Received for publication 20 May 1964. of urocanic acid. This metabolic precursor of FIGLU was found in increased quantities in $88 \%$ of a large number of urine samples from patients with various diseased states including megaloblastic anaemia. In $42 \%$ of the samples, urocanic acid was present in larger amounts than FIGLU itself. In fact, 10 specimens showed no FIGLU at all, despite a high content of urocanic acid. This was explained as being due to inhibition of urocanase by high tissue FIGLU, with subsequent build-up of urocanic acid levels. It was suggested that rising tissue levels of urocanic acid may then activate urocanase so that FIGLU reappears in the urine. Chanarin (1963) has recently cited a case showing the kind of alternating excretion of FIGLU and urocanic acid that one would expect on this hypothesis.

These facts make it desirable in clinical work to estimate both FIGLU and urocanic acid. The method devised for this purpose by Chanarin and Bennett (1962) requires the preparation of a crude liver enzyme. This is tedious and time-consuming, and in our hands, at least, has not been invariably successful. Moreover, as Cooperman and Luhby (1962) have pointed out, the value for urinary FIGLU is obtained indirectly by differential assays before and after removal of FIGLU or urocanic acid from the test sample. Even then, the estimated FIGLU may be artificially high due to variable additional amounts generated from other intermediates during the period of incubation with the relatively crude enzyme preparations used.

There remains, obviously, a need for a simpler and more rapid method for simultaneous detection and at least semi-quantitative determination of urinary 
FIGLU and urocanic acid in clinical practice. We believe that the method described below, using thin layer chromatography, meets these requirements, and is well within the capacities of a chemical laboratory attached to a general hospital. The method is comparable in sensitivity to others previously described, and can be adapted (by using standards) to semi-quantitative estimation of FIGLU and urocanic acid.

\section{METHODS}

MATERIALS Formimino-L-glutamic acid (FIGLU) was obtained as the barium salt from Sigma Chemical Co., St. Louis, Mo., U.S.A. (M.W. 259.7; Ba 27\%), and urocanic acid from L. Light \& Co. Ltd., Colnbrook, Bucks. Cellulose powder (MN-300G) for thin layer chromatography was obtained from Macherey, Nagel \& Co., Düren, Germany, A 'marker' urine containing 500 $\mu \mathrm{g}$. FIGLU per ml. was kindly supplied by Dr. J. Kohn, Queen Mary's Hospital, Roehampton.

THIN-LAYER CHROMATOGRAPHY This procedure was carried out with the Shandon equipment (basic outfit no. 2805). Glass plates of suitable size $(20 \times 20 \mathrm{~cm}$. $)$ were spread with a $200 \mu$ layer of cellulose powder MN-300 G, allowed to dry and then activated at $105^{\circ} \mathrm{C}$. for 30 minutes. The plates were briefly reactivated at $105^{\circ} \mathrm{C}$. for 10 minutes just before use to avoid the need for storage in a desiccator.

Urine, $5 \mu \mathrm{l}$., was applied to duplicate cellulose plates, and both were run for 2.5 hours in n-butanol:glacial acetic acid:water (114:38:60). One of the plates was dried in a fan-operated oven at $50^{\circ} \mathrm{C}$. for 25 minutes and then exposed to ammonia vapour in a tank (beaker of ammonia solution AnalaR sp. gr. 0.88 in the bottom of the tank) for 60 minutes in order to convert FIGLU to glutamic acid. The other (control) was left untreated, and allowed to dry in an atmosphere as ammonia-free as possible. The ammonia-treated plate was placed in the fan-operated oven for 10 to 15 minutes to drive off excess ammonia, then allowed to cool.

LOCATION OF COMPOUNDS Amino-acids were detected by spraying the plates with $0.2 \%$ ninhydrin (AnalaR) in acetone, a drop of pyridine being added to the reagent for increased sensitivity. FIGLU appeared as a purple spot on the ammonia-treated plate after decomposition to glutamic acid. The intensity of this spot was maximal after about one hour at room temperature. The appearance of the plate at one hour could be preserved by spraying with $0.25 \mathrm{M}$ nickel sulphate. Urocanic acid was revealed as an orange-red spot after spraying with Pauly reagent (Smith, 1960). The ammonia-treated plate was sprayed completely with ninhydrin solution, but we found it preferable, by use of an adjustable close-fitting perspex shield, to spray the lower half only of the control plate with ninhydrin. This left the upper half free for treatment with Pauly reagent. Improved visualization of the spots was obtained in this way. Dividing the plate into the appropriate 'lower' and 'upper' halves so as to separate FIGLU and urocanic acid clearly presented no problem, as their respective $R_{F}$ values differ widely (FIGLU: range 0.41 to 0.50 : urocanic acid range 0.68 to 0.73). We accepted a ninhydrin-positive spot as FIGLU when it appeared on the ammonia-treated plate at the appropriate place, but was absent on the control plate.

SEMI-QUANTITATION OF RESULTS This was performed by running on the same plates as the test urines two $2.5 \mu \mathrm{l}$. samples of normal urine to which had been added respectively $0.25 \mu \mathrm{g}$. FIGLU plus $0.125 \mu \mathrm{g}$. urocanic acid ('low standard') and 1.0 $\mu \mathrm{g}$. FIGLU plus $0.5 \mu \mathrm{g}$. urocanic acid ('high standard'); $2.5 \mu \mathrm{l}$. of the normal urine was also run as control. All normal urine samples had added in addition 1.25 $\mu \mathrm{g}$. glutamic acid $\left(R_{F}\right.$ range: 0.39 to 0.44 ) to aid in localization and identification of the FIGLU, since FIGLU appears as a discrete spot on the ammonia-treated plate just ahead of the glutamic acid. Glutamic acid, of course, appears on the control plate as well.

The use of glutamic acid as a marker substance is important, since, as is now well known, $R_{F}$ values in thin layer chromatography are not as constantly reproducible as with paper chromatography. A range of values for a given compound should be determined and quoted by each laboratory. The relative $R_{F}$ values, however, remain unchanged, and a test substance, e.g., FIGLU, can be readily identified by reference to a suitable marker, e.g., glutamic acid. These two substances always separate well, in spite of the overlapping $R_{F}$ ranges quoted above.

On one $20 \times 20 \mathrm{~cm}$. plate, it is possible to run the normal urine, the two standards, and up to six test urines. FIGLU and urocanic acid appearing in the test urines are roughly quantitated by visual comparison with the two standard urines. If the test urines contain amounts greater than the high standard, they are appropriately diluted to fall within the standard range and run again on fresh plates. Recoveries of FIGLU and urocanic acid added to test urines are satisfactory ( 80 to $106 \%$ ) in view of the semi-quantitative nature of the estimation.

CLINICAL Urine was collected over an eight-hour period after the patients had drunk $15 \mathrm{~g}$. L-histidine monohydrochloride (B.D.H. Ltd., Poole, Dorset; L. Light \& Co., Colnbrook, Bucks.) in $300 \mathrm{ml}$. of tepid water. (Some of the tests quoted in this paper were performed with a $5 \mathrm{~g}$. histidine load, owing to difficulty in obtaining supplies of the amino-acid.) The sample was preserved with $5 \mathrm{ml}$. N HCl plus a few crystals of thymol, and aliquots of the measured total volume stored at $-20^{\circ} \mathrm{C}$. until assayed.

\section{RESULTS}

SEPARATION OF FIGLU, UROCANIC ACID, AND SOME AMINO-ACIDS FROM AQUEOUS SOLUTION Some separations from aqueous solution were carried out in order to determine the range of sensitivities and $R_{F}$ values. In Fig. 2 FIGLU is seen to run well ahead of glutamic acid, glycine, histidine, and 


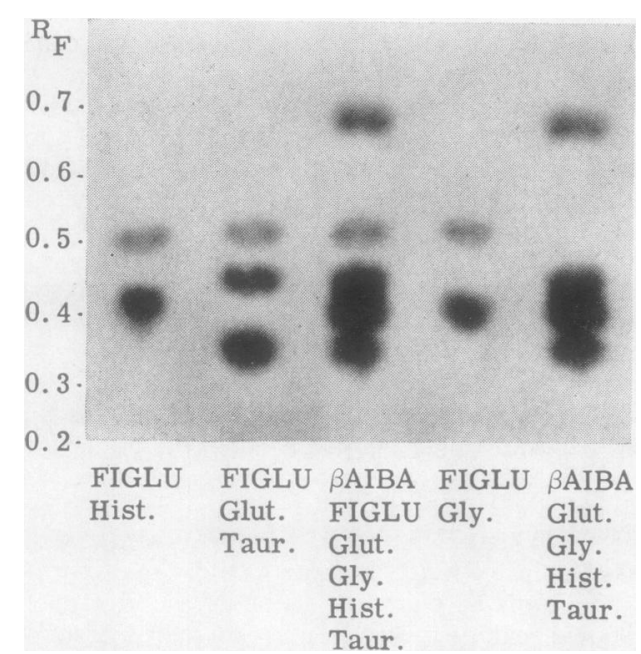

FIG. 2. Separation of five mixtures of amino-acids on a cellulose plate using n-butanol-acetic acid-water (114:38 : 60); $2.5 \mathrm{hr}$. run. FIGLU (formimino-glutamic acid, $\left.2.5 \mu g ., R_{F} 0.50\right) ;$ Hist. (histidine, $5 \mu g$., $R_{F} 0.41$ ); Glut. (glutamic acid, $\left.2.5 \mu g ., R_{F} 0.44\right) ;$ Taur. (taurine, $5 \mu g$., $\left.R_{F} 0.35\right) ; \beta A I B A$ ( $\beta$-aminoisobutyric acid, $5 \mu g ., R_{F}$ 0.64); Gly. (glycine, $5 \mu g$., $R_{F} 0 \cdot 40$ ). taurine. $\beta$-Aminoisobutyric acid runs well in advance of FIGLU. Generalised aminoaciduria and increased urinary excretion of taurine have been reported to occur in patients with megaloblastic anaemias, especially those due to vitamin $B_{12}$ deficiency (Neill and Weaver, 1958; Todd, 1959). Histidine, of course, appears in large amounts after loading with this amino-acid. Although these amino-acids do not all separate well from each other, except glutamic acid, taurine, and $\beta$-aminoisobutyric acid, this does not matter; the important fact is that they do not interfere with FIGLU. Urocanic acid ran ahead of all these amino-acids and could be detected with Pauly reagent $\left(R_{F} 0 \cdot 68\right)$. Using serial dilutions of the standard substances, FIGLU could be detected down to $0.125 \mu \mathrm{g}$. and urocanic acid down to $0.0625 \mu \mathrm{g}$.

SEPARATION AND DETECTION OF FIGLU AND UROCANIC ACID ADDED TO NORMAL URINE When added to normal urine, FIGLU and urocanic acid can be detected in roughly the same amounts as from aqueous solutions. In most urines tested (normal and pathological) there is a trace of a ninhydrin-reacting substance where FIGLU would be expected to appear after ammonia treatment. This contaminant

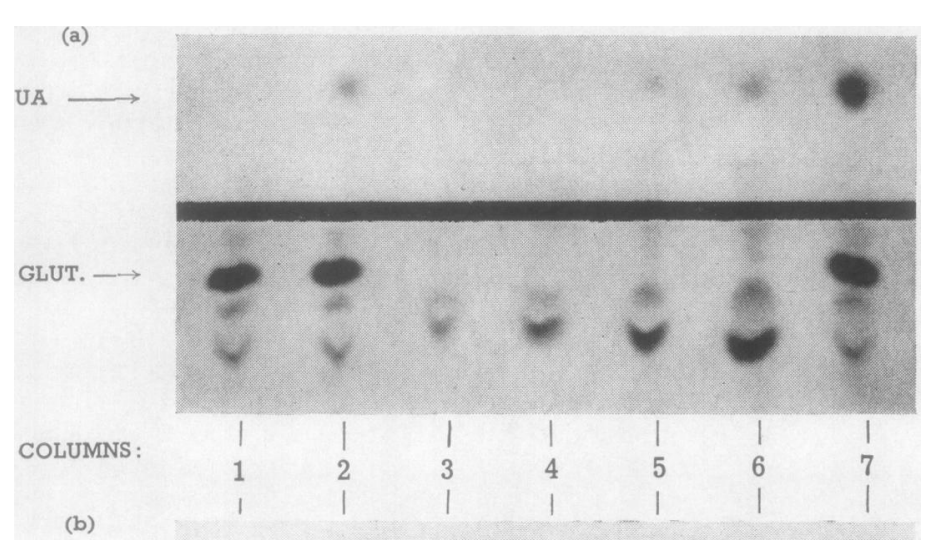

(b)
FIG. 3. Control plate (above) and ammonia-treated plate (below).

Photograph of serial dilutions of a

'marker urine' containing $500 \mu \mathrm{g}$.

FIGLU/ml. run against standards.

(Standards here contain twice the

amounts of glutamic acid, FIGLU, and urocanic acid routinely used in order to improve the photographic reproduction.) Column 1: 2.5 $\mu$ l. normal urine; $2.5 \mu \mathrm{g}$. glutamic acid (Glut.)

Column 2: 2.5 $\mu$ l. normal urine; $2.5 \mu \mathrm{g}$.

Glut.; 0.5 $\mu$ g. FIGLU; 0.25 $\mu$ g. urocanic $\operatorname{acid}(U A)$.

Column 3: $2.5 \mu l$. of $a 1$ in 3 dilution of marker urine.

Column 4: $5 \mu l$. of a 1 in 3 dilution of marker urine.

Column 5: 2.5 $\mu l$. marker urine.

Column 6: $5 \mu l$. marker urine.

Column 7: 2.5 $\mu \mathrm{l}$. normal urine; $2.5 \mu \mathrm{g}$.

Glut.; $2.0 \mu g$. FIGLU; $1.0 \mu g$. urocanic acid.

The dilutions and amounts of marker urine spotted (columns 3-6) contained quantities of $0 \cdot 4,0.8,1 \cdot 25$, and $2.5 \mu \mathrm{g}$. FIGLU respectively. Note (Fig. $3 b$ ) the serial gradation in the intensities of the FIGLU spots, which should be compared with the spots from $0.5 \mu \mathrm{g}$. FIGLU and $2 \mu \mathrm{g}$. FIGLU. Note the presence of urocanic acid in the marker urine (Fig. 3a). 
however, occurs on both plates (control and ammonia-treated) and is rather fuzzy in outline whereas FIGLU itself forms a much sharper and better-defined spot. Although present in small amounts as a rule, it may reduce the accuracy of quantitation of FIGLU in urines in which FIGLU occurs in low concentration.

Urocanic acid spots have never been observed to be contaminated with other Pauly-reacting substances; they appear in a clear field and quantitative estimation by visual comparison with the standards is easier than with the FIGLU.

FIGLU AND UROCANIC ACID IN 'MARKER' URINE Figures $3 a$ and $3 b$ show a series of dilutions of a 'marker' urine containing $500 \mu \mathrm{g}$. FIGLU/ml., compared with suitable standards. The depth of the FIGLU spots in the marker urine corresponds quite well with the amounts of FIGLU expected when compared with the standards. The marker urine dilutions also show an excellent gradation in the density of the FIGLU spots. Spraying with Pauly reagent (Fig. 3a) shows that the marker urine also contains urocanic acid, the estimated concentration from comparison with the standards being about $50 \mu \mathrm{g} . / \mathrm{ml}$.

TEST URINES Table I summarizes the results of the urinary excretion of histidine metabolites after a histidine load of 5 to $15 \mathrm{~g}$. in eight normal subjects, five patients with megaloblastic anaemia due to vitamin $B_{12}$ and/or folic acid deficiency, and a patient with erythraemic myelosis.

Case 9 is of particular interest in the light of the recent report (Forshaw, Harwood, and Weatherall, 1964) of folic acid deficiency in patients with myelofibrosis. A diagnosis of pernicious anaemia had been made in 1952 on the basis of peripheral blood findings of a macrocytic anaemia. From 1955 onwards she showed a moderate persistent leucocytosis, thrombocythaemia, and hepato-splenomegaly with biopsy evidence of extramedullary haemopoiesis in the liver. Parenteral vitamin $\mathbf{B}_{12}$ therapy had been given from 1952 to 1959. She presented again in 1963 with a megaloblastic anaemia, histamine-fast achlorhydria, an absorptive defect of vitamin $B_{12}$ of pernicious anaemia type, and a serum vitamin $B_{12}$ level of 136 and $144 \mu \mu \mathrm{g}$./ $\mathrm{ml}$. (normal 150-800 $\mu \mu \mathrm{g}$./ml.). The histidine load test was carried out seven days after the start of a daily regimen of $1,000 \mu \mathrm{g}$. of vitamin $\mathrm{B}_{12}$ parenterally.

Case 10 with pernicious anaemia showed a satisfactory haematological response to vitamin $\mathbf{B}_{12}$ and the serial reduction in the amount of abnormal histidine metabolites followed the pattern previously described (Chanarin, 1963). Cases 11 and 12 were treated with vitamin $B_{12}$ and folic acid, the former having shown a suboptimal response to vitamin $B_{12}$ alone and the latter to folic acid alone. Malabsorption of vitamin $\mathrm{B}_{12}$ was demonstrated in both cases and in neither was it corrected on repeating the tests with hog intrinsic factor. Case 13 showed a full haematological response to folic acid while his anticonvulsants were continued.

Case 14, with erythraemic myelosis, studied during a phase of increased transfusion requirements, in the absence of evidence of haemolysis, is of consider-

TABLE I

URINARY EXCRETION OF HISTIDINE METABOLITES IN EIGHT NORMALS AND SIX PATIENTS

\begin{tabular}{|c|c|c|c|c|c|c|c|}
\hline \multirow[t]{2}{*}{ No. } & \multirow[t]{2}{*}{ Age (yr.) } & \multirow[t]{2}{*}{$\operatorname{Sex}$} & \multirow[t]{2}{*}{ Diagnosis } & \multirow{2}{*}{$\begin{array}{l}\text { Histidine } \\
\text { Load }(g .)\end{array}$} & \multicolumn{3}{|c|}{ Histidine Metabolites (mg./8 hours) ${ }^{1}$} \\
\hline & & & & & FIGLU & $\begin{array}{c}\text { Urocanic } \\
\text { Acid }\end{array}$ & Total \\
\hline 1 & 33 & $\mathbf{M}$ & Normal (staff) & 15 & 0 & 5 & 5 \\
\hline 2 & 24 & $\mathbf{F}$ & Normal (staff) & 15 & 10 & 10 & 20 \\
\hline 3 & 44 & $\mathbf{M}$ & Normal (staff) & 15 & 0 & 5 & 5 \\
\hline 4 & 17 & $\mathbf{F}$ & Normal (staff) & 15 & 5 & 5 & 10 \\
\hline 5 & 40 & $\mathbf{M}$ & Normal (staff) & 15 & 0 & 5 & 5 \\
\hline 6 & 21 & $\mathbf{F}$ & Normal (staff) & 15 & 5 & 5 & 10 \\
\hline 7 & 30 & $\mathbf{M}$ & Normal (staff) & 15 & 0 & 5 & 5 \\
\hline 8 & 23 & $\mathbf{F}$ & Normal (staff) & 15 & 15 & 20 & 35 \\
\hline 9 & 54 & $\mathbf{F}$ & Myeloproliferative syndrome with pernicious anaemia & 15 & 120 & 60 & 180 \\
\hline 10 & 77 & $\mathbf{F}$ & Pernicious anaemia & 15 & 140 & 70 & 210 \\
\hline & & & $-B_{12} \times 3$ days & 15 & 90 & 10 & 100 \\
\hline & & & $-B_{18} \times 8$ days & 15 & 25 & 0 & 25 \\
\hline 11 & 63 & $\mathbf{M}$ & Idiopathic steatorrhoea with megaloblastic anaemia & 5 & 350 & 15 & 365 \\
\hline 12 & 53 & $\mathbf{F}$ & Idiopathic steatorrhoea with megaloblastic anaemia & 5 & 140 & 50 & 190 \\
\hline 13 & 41 & $\mathbf{M}$ & Megaloblastic anaemia due to phenobarbitone and primidone & 5 & 40 & $\mathbf{0}$ & 40 \\
\hline 14 & 72 & $\mathbf{M}$ & Erythraemic myelosis & 15 & 25 & 950 & 975 \\
\hline
\end{tabular}

${ }^{1}$ In view of the semi-quantitative nature of the estimation, the calculated total excretion is expressed as follows:-

3-5 mg. as $5 \mathrm{mg}$., 6-50 mg. to nearest $5 \mathrm{mg}$., $51-100 \mathrm{mg}$. to nearest $10 \mathrm{mg}$., 101-200 mg. to nearest $20 \mathrm{mg}$., $>200 \mathrm{mg}$. to nearest $50 \mathrm{mg}$. Excretions less than $2 \mathrm{mg}$. $/ 8 \mathrm{hrs}$. were not usually detectable by this method. 
able interest in the amounts of abnormal histidine metabolites excreted by this patient. Objective evidence of folic acid deficiency has not, to our knowledge, previously been described in erythraemic myelosis, although theoretically such a deficiency might be expected considering the hyperplastic, though deranged and ineffective, erythropoiesis that is the hallmark of this disease. During our 18-month period of observation serum vitamin $B_{12}$ levels and radio-cobalt vitamin $B_{12}$ absorption tests were all normal on three separate occasions, the last series of tests having been carried out at the time of the histidine load tests. It should be noted that the abnormal excretion in this patient is accounted for almost exclusively by urocanic acid. Marked improvement in the excretory pattern occurred with $15 \mathrm{mg}$. folic acid daily by mouth; complete reversal to normal, however, was not obtained until the same dose was given by intramuscular injections on each of three successive days. This case is shortly to be reported in greater detail elsewhere.

\section{DISCUSSION}

The method described using thin-layer chromatography for the simultaneous separation and detection from urine of FIGLU and urocanic acid has proved to be relatively simple, consistent, and reproducible. FIGLU down to $0.125 \mu \mathrm{g}$. and urocanic acid down to $0.0625 \mu \mathrm{g}$. can be detected, and the amounts present in urine can be roughly quantitated by comparison with standards run at the same time. Thin-layer chromatography, now very widely used for rapid separations, is a simpler process to carry out than high voltage electrophoresis, and the sensitivity is just as good, if not better, with the former technique. Enzymatic techniques are, of course, more sensitive, but are difficult and take much longer to carry out.

The new technique has been applied very successfully to many normal and abnormal urines and the results in six representative cases are briefly described. A feature of the method is that FIGLU and urocanic acid can be estimated simultaneously. If, as claimed by Bennett and Chanarin (1962), it is the total of these two metabolites which is the clinically significant figure, this is an important advantage of the technique.

The main disadvantage of the method as it stands is the lack of precise quantitation. It is hoped to develop the method into one more strictly quantitative by eluting the FIGLU and urocanic acid from the cellulose and measuring their coloured derivatives spectrophotometrically. For general screening purposes and for following the results of specific therapy, rough quantitation by visual inspection of the spots against standards seems to be satisfactory.

We wish to thank Professor A. G. Macgregor, Drs. J. Crooks, K. N. V. Palmer, and L. Wilson, and Mr. C. G. Clark for access to cases under their care; and Sisters D. R. Thomson and J. V. Sinclair and the nursing staff of ward 4, A.R.I., for the metabolic collections. Our thanks are also due to Mr. W. Topp and Miss Mary Martin for help with the figures. The intrinsic factor preparation used for the vitamin $\mathbf{B}_{\mathbf{1 2}}$ absorption studies was a gift from Dr. P. M. Worrall, Lederle Laboratories, London.

\section{REFERENCES}

Bakerman, H. A., Silverman, M., and Daft, F. S. (1951). J. biol. Chem., $188,117$.

Bennett, M. C., and Chanarin, I. (1962). Nature (Lond.), 196, 271.

Borek, B. A., and Waelsch, H. (1953). J. biol. Chem., 205, 459.

Chanarin, I. (1963). Brit. J. Haemat., 9, 141.

C, and Bennett, M. C. (1962). Brit. med. J., 1, 27.

Cooperman, J. M., and Luhby, A. L. (1962). Jbid., 1, 1072.

Forshaw, J., Harwood, L., and Weatherall, D. J. (1964). Brit. med. J., 1,671 .

Knowles, J. P., Prankerd, T. A. J., and Westall, R. G. (1960). Lancet, 2, 347.

Kohn, J., Mollin, D. L., and Rosenbach, L. M. (1961). J. clin. Path., $14,345$.

Luhby, A. L., Cooperman, J. M., and Teller, D. N. (1959a). Amer. J. clin. Nutr., 7, 397.

,$- \longrightarrow$ (1959b). Proc. Soc. exp. Biol. (N.Y.), 101, 350.

Neill, D. W., and Weaver, J. A. (1958). Brit. J. Haemat., 4, 447.

Silverman, M., Gardiner, R. C., and Condit, P. T. (1958). J. nat. Cancer Inst., 20, 71.

Smith, I. (1960). Chromatographic and Electrophoretic Techniques, vol. I, page 218. Heinemann, London.

Tabor, H., Silverman, M., Mehler, A. H., Daft, F. S., and Bauer. H. (1953). J. Amer. chem. Soc., 75, 756.

- - and Wyngarden, L. (1958). J. clin. Invest., 37, 824.

Todd, D. (1959). J. clin. Path., 12, 238. 